\title{
Washing increases the susceptibility to exogenous oxidative stress in red deer spermatozoa
}

\author{
Á.E. Domínguez-Rebolledo ${ }^{a}$, M.R. Fernández-Santos ${ }^{\text {b }}$, O. García-Álvarez ${ }^{\text {b }}$, \\ A. Maroto-Morales ${ }^{\mathrm{a}}$, J.J. Garde ${ }^{\mathrm{a}}$, F. Martínez-Pastor ${ }^{\mathrm{a}, \mathrm{c}, *}$ \\ ${ }^{a}$ Biology of Reproduction Group, National Wildlife Research Institute (IREC) (UCLM-CSIC-JCCM), 02071 Albacete, Spain \\ ${ }^{\mathrm{b}}$ Regional Center of Animal Selection and Reproduction (CERSYRA), JCCM, Valdepeñas, 13300 Ciudad Real, Spain \\ ${ }^{\mathrm{c}}$ ITRA-ULE, INDEGSAL, University of León, 24071 León, Spain
}

Received 3 April 2009; received in revised form 1 June 2009; accepted 3 June 2009

\begin{abstract}
The effects of routine sperm work are often overlooked. We assessed the effect of washing cryopreserved epididymal spermatozoa from red deer (Cervus elaphus hispanicus, Helzheimer 1909). After thawing, epididymal samples (four stags) were diluted in TALP-HEPES. A split was left untouched, another was centrifuged $(300 \times \mathrm{g}, 5 \mathrm{~min})$ and resuspended, and a third was centrifuged and the supernatant substituted by fresh TALP-HEPES (washing). Each split was supplemented either with nothing, $1 \mathrm{mM}$ of the antioxidant Trolox, $100 \mu \mathrm{M}$ of the oxidant Fe (with ascorbate), or both. The $3 \times 4$ treatments were incubated at $37 \mathrm{C}$ and assessed each hour up to $3 \mathrm{~h}$ for motility (computer-aided sperm assessment) and viability/apoptosis plus mitochondrial status (YO-PRO-1, propidium iodide, Mitotracker Deep Red; flow cytometry). DNA damage at $4 \mathrm{~h}$ was assessed using the terminal deoxynucleotidyl transferase-mediated dUTP nick end-labeling assay. Centrifugation alone affected neither sperm quality nor DNA, and the oxidant had no effect in control or centrifuged samples. Washed samples were not different than control, but oxidant decreased motility, mitochondrial status and viability, and altered the motility subpopulation pattern, being partially suppressed by Trolox. Spermatozoa with damaged DNA dramatically increased in the washed-oxidized sample (from $22.30 \pm 3.52 \%$ to $67.94 \pm 5.07 \%$ ), but not when antioxidant was present. Although samples from different males behaved similarly, male-to-male variability was detected regarding susceptibility to oxidative damage after washing. We concluded that, although red deer thawed spermatozoa seemed resilient to centrifugation, the vulnerability to oxidative stress after washing makes it advisable to supplement manipulation media with antioxidants, especially taking into account male-to-male variability.
\end{abstract}

(C) 2009 Published by Elsevier Inc.

Keywords: Antioxidants; Centrifugation; Oxidative stress; Red deer; Washing

\section{Introduction}

Sperm washing is a common procedure in assisted reproduction. During cryopreservation, in vitro fertilization, or other procedures, spermatozoa must be placed in different media, and the simplest method involves

\footnotetext{
Q1

* Corresponding author.

E-mail address: fmarp@unileon.es (F. Martínez-Pastor).
}

centrifugation, removal of the supernatant, and resuspension in the new media. Although this technique has been perfected (e.g., using discontinuous density gradients), simple washing remains as a basic step in many protocols, being routinely used to remove seminal plasma from semen or the cryopreserving extender from thawed spermatozoa [1-4].

However, it is evident that centrifugal forces exert mechanical stress on spermatozoa, which might reflect into a lower quality or a decreased ability to sustain 
other kinds of stress [5,6]. It has been shown that spermatozoa from many species are sensitive to mechanical stress, such as the human spermatozoon $[5,7,8]$, but remarkably also rodent spermatozoa, whose long flagella and special morphology makes them very vulnerable to mechanical stress $[9,10]$. Compared with spermatozoa of rodents, ruminants and boar spermatozoa seem to be much less susceptible to damage by mechanical stress [10]. Moreover, although spermatozoa from these species could be affected by centrifugation, any negative consequences are often more than compensated by the positive effects of washing $[6,11]$.

The effects of centrifugation possibly extends beyond the direct action of shear forces and close packing of spermatozoa. Aitken and Clarkson [12] demonstrated that centrifugal pelleting of unselected human sperm populations caused a burst in the generation of reactive oxygen species (ROS) within the pellet, which induced irreversible damage to spermatozoa and the impairment of their in vitro fertilizing ability. Other authors confirmed the link among centrifugation and oxidative damage, reporting that repetitive washing cycles, by means of resuspension-centrifugation, caused a 20 - to 50 -fold increase in the levels of ROS in human semen [13]. Twigg et al. [14] reported that a previous washing step increased the oxidative damage after swim-up, highlighting the negative impact in DNA integrity. In fact, relatively harmless procedures involving mechanical agitation have been related to the generation of ROS in human semen after liquefaction [15] (whereas they seem to have no effect in other species [16]). Furthermore, the addition of antioxidants have helped to ameliorate the damage caused to human spermatozoa by centrifugation [17-19], indicating that, at least in some species, ROS are responsible for centrifugation damage.

In this study, we concentrated on the exploration of the effect of free radicals in washed spermatozoa obtained from the epididymides of red deer. There have been many advancements in the application of artificial reproductive techniques in red deer, due to the interest as domestic and game species [20,21]. Our group has contributed with the development of cryopreservation protocols for postmortem samples [22-26] and for semen obtained by electroejaculation $[27,28]$. However, some techniques have been overlooked, and its improvement could benefit the outcome of current protocols. We have noticed that deer spermatozoa are fairly resilient to centrifugation stress (as seem to be other ungulates [10]), but we are concerned that this technique, especially after thawing, might decrease sperm quality. Because most studies on the effect of washing on sperm quality and its remediation have been carried out in human semen, and being aware of the differences between species, we have approached this topic in red deer semen. Therefore, we have tested several hypothesis on red deer thawed spermatozoa: if washing causes a loss of sperm quality; if washing causes a higher vulnerability to oxidative damage; and, consequently, if antioxidant supplementation could remediate that damage. Moreover, we aimed at differentiating the centrifugation step from the whole washing procedure to test if the damage resulting from the technique would be due to the pelleting (i.e., to the centrifugation per se) or if post-pelleting medium removal and resuspension could have a role. Finally, because male-to-male variability often noticeably influences sperm characteristics [29], we have studied if the response to different males to the treatments was alike.

\section{Materials and methods}

\subsection{Reagents and media}

Fluorescence probes and the ApoTarget APOBRDU Kit (terminal deoxynucleotidyl transferasemediated dUTP nick end-labeling [TUNEL] test) were purchased from Invitrogen (Barcelona, Spain). Flow cytometry equipment, software, and consumables (including the sheath fluid) were purchased from BD Biosciences (San José, CA, USA). Preparation and staining of samples for flow cytometry analysis were performed using flow cytometry PBS (BD FACSFlow; Q2 129 BD Biosciences). The rest of the chemicals (reagent 130 grade or higher) were acquired from Sigma (Madrid, Spain). Stock solutions of the antioxidant and the oxidant solution were prepared as $500 \mathrm{mM}$ Trolox (vitamin $\mathrm{E}$ analogue) in ethanol and as $10 \mathrm{mM}$ FeSO and $50 \mathrm{mM}$ sodium ascorbate (Fe/ascorbate) in water, respectively. Stock solutions of the fluorescence probes were as follows: Hoechst 33342, $5 \mathrm{mg} / \mathrm{mL}$ in water; propidium iodide (PI), $7.5 \mathrm{mM}$ in water; YO-PRO-1, $50 \mu \mathrm{M}$ in dimethyl sulfoxide (DMSO); Mitotracker Deep Red, $1 \mathrm{mM}$ in DMSO. All solutions were kept at$20 \mathrm{C}$ (except Hoechst 33342, which was kept at 5 C) and in the dark until needed, except for the Trolox and the oxidant solutions, which were prepared the same day. TALP-HEPES was composed of: $87 \mathrm{mM} \mathrm{NaCl,Q3} 144$ $3.1 \mathrm{mM} \mathrm{KCl}, 2 \mathrm{mM} \mathrm{CaCl}, 0.4 \mathrm{mM} \mathrm{MgCl}, 0.3 \mathrm{mM} \quad 145$ NaHPO, $40 \mathrm{mM}$ HEPES, $21.6 \mathrm{mM}$ sodium lactate, 146 $1 \mathrm{mM}$ sodium pyruvate, $50 \mu \mathrm{g} / \mathrm{mL}$ kanamycin, $10 \mu \mathrm{g} / \quad 147$ $\mathrm{mL}$ phenol red, and $6 \mathrm{mg} / \mathrm{mL}$ bovine serum albumin (BSA) (pH 7.5). 

198 Q4 The experiment was triplicated within each male.

\subsection{Animals and sperm cryopreservation} minimum period of $1 \mathrm{yr}$ in liquid nitrogen.

\subsection{Experimental design} medium).
Epididymal samples were collected from four mature stags (Cervus elaphus hispanicus, Helzheimer 1909) that were legally culled and hunted in their natural habitat during the rutting season (September to October). Hunting was in accordance with the harvest plan of game reserves, following Spanish Harvest Regulation (Law 2/93 of Castilla-La Mancha), which conforms to European Union regulations. These operations were carried out as part of a project approved by the ethical committee of the University of CastillaLa Mancha and that adheres to the Guidelines for the Care and Use of Animals. Spermatozoa were collected from the cauda epididymis within $3 \mathrm{~h}$ postmortem and diluted at ambient temperature to $200 \times 10$ spermato$\mathrm{zoa} / \mathrm{mL}$ in Triladyl (Minitüb, Tifenbach, Germany) with $20 \%$ egg yolk and $3 \%$ glycerol. Extended spermatozoa were cooled down to $5 \mathrm{C}(-2 \mathrm{C} / \mathrm{min})$ and equilibrated for $2 \mathrm{~h}$ at the same temperature. Samples were loaded into $0.25-\mathrm{mL}$ plastic straws (IMV, L'Aigle Cedex, France) and frozen in nitrogen vapor $(4 \mathrm{~cm}$ above liquid nitrogen) for $10 \mathrm{~min}$. The straws remained for a

Samples were thawed by immersing straws in a water bath at $37 \mathrm{C}$ for $30 \mathrm{sec}$. Contents were poured into 1.5-mL microtubes, and, after $10 \mathrm{~min}$, spermatozoa were diluted down to $30 \times 10 \mathrm{~mL}$ using TALP-HEPES. The sperm suspension was split among three tubes, and two of them were centrifuged at $300 \times \mathrm{g}$ for $5 \mathrm{~min}$. The supernatant of one of the centrifuged tubes was removed and replaced by fresh TALP-HEPES (washing), and both pellets were gently resuspended. These three suspensions were termed Control, Centrifuged (centrifuged and resuspended in the same medium), and Washed (centrifuged and pellet resuspended in fresh

The content of each tube was divided among four tubes, adding (1) nothing; (2) $1 \mathrm{mM}$ Trolox; (3) $100 \mu \mathrm{M}$ $\mathrm{Fe} / 1 \mathrm{mM}$ ascorbate (oxidant); (4) $1 \mathrm{mM}$ Trolox and oxidant. The 12 tubes (three manipulations, four supplements) were incubated at $37 \mathrm{C}$ and assessed at $0,1,2$, and $3 \mathrm{~h}$ of incubation. The analyses performed at these times were motility (using computer-aided sperm assessment; CASA) and physiologic parameters using flow cytometry: viability, "apoptosis," and mitochondrial status. After $4 \mathrm{~h}$ of incubation, samples were fixed and processed for assessing DNA status (TUNEL test). stress in red deer spermatozoa. Theriogenology (2009), doi:10.1016/j.theriogenology.2009.06.027
Sperm were diluted down to $10 \times 10$ to $20 \times 10$ spermatozoa/mL and loaded into a Makler counting chamber $(10 \mu \mathrm{m}$ depth $)$ at $37 \mathrm{C}$. The CASA system consisted of a triocular optical phase-contrast microscope (Nikon Eclipse 80i; Nikon, Tokyo, Japan) equipped with a warming stage at $37 \mathrm{C}$ and a Basler A302fs digital camera (Basler Vision Technologies, Ahrensburg, Germany). The camera was connected to a computer by an IEEE 1394 interface. Images were captured and analyzed using the Sperm Class Analyzer (SCA2002) software (Microptic S.L., Barcelona, Spain). Sampling was carried out using a $\times 10$ negative phase-contrast objective (no intermediate magnification). Image sequences were saved and analyzed afterward. Software settings were adjusted to deer spermatozoa. The standard parameter settings were as follows: 25 frames/sec; 20 to $90 \mu \mathrm{m}$ for head area; VCL $>10 \mu \mathrm{m} / \mathrm{sec}$ to classify a spermatozoon as motile. For each spermatozoa, the software rendered the percentage of motile spermatozoa, three velocity parameters (VCL, velocity according to the actual path; VSL, velocity according to the straight path; VAP, velocity according to the smoothed path), three track linearity parameters (LIN, linearity; STR, straightness: WOB, wobble), the ALH (amplitude of the lateral displacement of the sperm head), and the BCF (head beat-cross frequency). These parameters have been defined elsewhere [30].

\subsection{Fluorescence probes}

Samples were diluted down to 10 spermatozoa $/ \mathrm{mL}$ in flow cytometry PBS with $5 \mu \mathrm{M}$ Hoechst 33342, $0.1 \mu \mathrm{M}$ YO-PRO-1, $10 \mu \mathrm{M}$ PI, and 0.1 $\mu \mathrm{M}$ Mitotracker Deep Red. After $20 \mathrm{~min}$ in the dark, the samples were run through a flow cytometer. YO-PRO-1 is a probe capable of staining early apoptotic cells (with intact plasmalemma, not stained by PI, but showing increased permeability), whereas Mitotracker Deep Red labels active mitochondria (with high mitochondrial membrane potential, ) [31]. The membrane-impermeant PI was used to identify spermatozoa with damaged plasmalemma, and Hoechst 33342 stained all nuclei, aiding in discarding noncellular debris from the analysis.

\subsection{TUNEL assay}

We used the TUNEL method to assess the presence of spermatozoa with nuclear DNA strand breaks following the manufacturer's instructions (ApoTarget

\subsection{CASA analysis}


APO-BRDU Kit; Invitrogen, Barcelona, Spain). Q5Briefly, spermatozoa diluted in PBS (10 mill./mL) were fixed for $1 \mathrm{~h}$ in $2 \%$ paraformaldehyde. The cells were washed with PBS, pelleted, and resuspended with ethanol at $0{ }^{\circ} \mathrm{C}$. The samples were left at $-20{ }^{\circ} \mathrm{C}$ overnight. Then, the cells were washed twice using the wash buffer provided with the kit, adding the DNA labeling mixture after removing the wash buffer. After $60 \mathrm{~min}$ at $37{ }^{\circ} \mathrm{C}$ (with agitation), the cells were washed twice using the rinse buffer. Finally, the cells were resuspended in the antibody solution (FITCAnti-BrdUTP $\mathrm{mAb}$ ) and incubated for $30 \mathrm{~min}$ at room temperature in the dark. Samples were resuspended in a PI/RNase A solution and analyzed by flow cytometry within $2 \mathrm{~h}$. Positive control (incubation of fixed cells with DNase A) and negative control (substituting water for the DNA labeling mixture) were used to standardize the assay.

\subsection{Flow cytometry analyses}

The flow cytometer was a Becton Dickinson LSR-I model (BD Biosciences, San José, CA, USA) furnished with a 325-nm He-Cd laser (excitation for Hoechst 33342), a 488-nm Ar-ion laser (excitation for YO-PRO1, PI, and TUNEL), and a 633-nm He-Ne laser (excitation for Mitotracker Deep Red). Fluorescence from Hoechst 33342 was read with the FL5 photodetector (424/44BP filter), YO-PRO-1 and TUNEL (fluorescein isothiocyanate [FITC]) were read with the FL1 photodetector (530/28BP filter), PI was read with the FL3 photodetector (670LP filter), and Mitotracker Deep Red was read with the FL6 photodetector (670/ Q6 40BP filter). FSC/SSC signals and Hoechst fluorescence were used to discriminate spermatozoa from debris. Fluorescence captures were controlled using the Cell Quest Pro 3.1 software (BD Biosciences). All the parameters were read using logarithmic amplification. For each sample, 5000 spermatozoa from each sample were recorded, saving the data in flow cytometry standard (FCS) v. 2 files. The analysis of the flow cytometry data was carried out using WEASEL v. 2.6 (WEHI, Melbourne, Australia). The YO-PRO-1/PI/ Mitotracker Deep Red staining was analyzed as previously described for red deer [31]. For analyzing sperm viability and "apoptosis," three subpopulations were identified: viable (YO-PRO-1-/PI-), "apoptotic" (YO-PRO-1 + /PI-), and membrane damaged $(\mathrm{PI}+)$; for analyzing mitochondrial status, the YO-PRO-1+ events were gated out before assessing the percentage of spermatozoa with high mitochondrial membrane potential (Mitotracker + ), therefore using only the viable-nonapoptotic subpopulation. We had confirmed $[31,32]$ that any YO-PRO-1+ spermatozoa have high mitochondrial membrane potential (). Thus, we deemed it appropriate to consider only the YO-PRO-1subpopulation for studying mitochondrial status, preventing the confounding effect of varying "apoptotic" or "necrotic" subpopulations. For TUNEL analysis, the negative control allowed us to define the TUNELpopulation, thus events with increased fluorescence were counted as TUNEL+ cells.

\subsection{Statistical analysis}

For the statistical analysis, we used the R statistical environment [33] (results are shown as means and standard errors). To analyze the effects of time, processing (none, centrifugation, or washing) and supplement (none, oxidant, and/or antioxidant) on sperm parameters, we used linear mixed-effects models, nesting replicates within males and using them as the random part of the models. In a second analysis, we evaluated the between-male variability regarding the response to the treatments. Moreover, to disclose the subpopulation structure of each sample according to motility characteristics for each spermatozoa, CASA data was processed by cluster analysis. Briefly, we first chose VAP, STR, ALH, and BCF as the most informative parameters (minimizing correlations). Spermatozoa were initially grouped by using a nonhierarchical method (k-means; CLARA algorithm) to produce 25 homogeneous clusters. These clusters were grouped via a model-based clustering (hierarchical method) using the Bayesian Information Criterion (BIC) to find the optimal model and final number of clusters. Then, the proportion of each cluster in each sampling was studied in the same manner as the rest of the parameters.

\section{Results}

In general, any of the processing treatments had a dramatic effect in sperm quality, but there were important interactions among the effects of the supplements and the processing. Sperm motility (Fig. 1) decreased with time in all cases $(\mathrm{P}<0.001)$, and we could detect a significant negative effect of washing (overall effect:-9.45 $\pm 3.97, \mathrm{P}=0.018$ ), especially when the sample was supplemented with the oxidant (effect: $-24.24 \pm 5.65, \mathrm{P}<0.001)$. Trolox had a positive overall effect $(\mathrm{P}<0.043)$ and could prevent the oxidant effect on washed samples $(\mathrm{P}<0.024)$, but not totally. Kinematic parameters (Fig. 2) were affected 


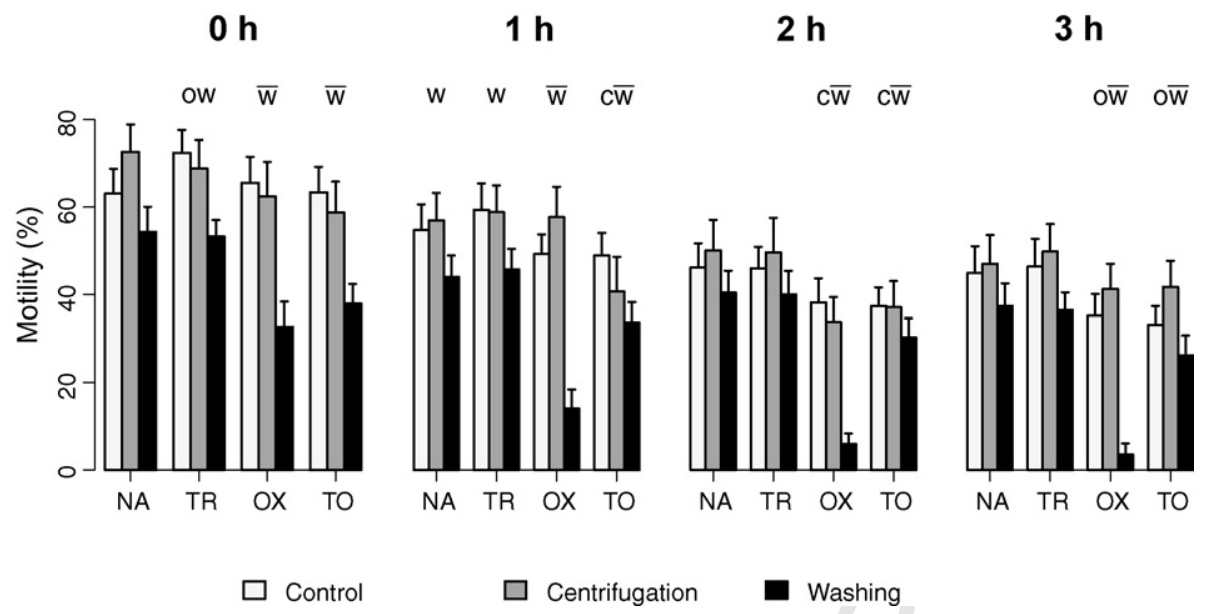

Fig. 1. Sperm motility (mean $\pm \mathrm{SEM}$ ) at the four sampling times. Means are shown for each supplement and treatment (NA, no additive; TR, 1 mM Trolox; OX, $100 \mu \mathrm{M} \mathrm{Fe} / 1 \mathrm{mM}$ ascorbate; TO, TR and OX). The letters indicate significant differences $(\mathrm{P}<0.05)$ within each time between the treatment Control/NA (first column on the left of each graph) and Control (o), Centrifuged (c), or Washing (w) treatments in each supplement group. An overbar indicates $\mathrm{P}<0.001$.

differently. Velocity (VCL, VAP, VSL; P $<0.001$ ) and ALH $(P=0.009)$ decreased slowly with time and, although washing had no significant effect by itself, it decreased these parameters when combined with the oxidant $(\mathrm{P}<0.008)$, and this effect increased with time regarding velocities $(\mathrm{P}<0.001)$. On the oxidantstressed samples, Trolox reverted the effect of the oxidant only for VCL $(\mathrm{P}=0.002)$ and ALH $(\mathrm{P}=0.001)$ and reduced the rate at which the three velocity parameters decreased with time $(\mathrm{P}<0.05)$. Head beatcross frequency (BCF) declined with time only in washed samples submitted to oxidative stress ($0.57 \pm 0.22$ per hour, $\mathrm{P}=0.010)$, and, again, Trolox cancelled that effect $(\mathrm{P}=0.031)$.

Clustering the motility data yielded three subpopulations (discarding nonmotile spermatozoa). Cluster 1 grouped slow spermatozoa, Cluster 2 grouped fast and linear spermatozoa, and Cluster 3 grouped fast but erratic spermatozoa (see Table 1). The initial proportions of each subpopulation (in the Control without supplementation) were $44.26 \pm 4.63 \%$ for Cluster 1 , $43.95 \pm 3.57 \%$ for Cluster 2 , and $12.95 \pm 2.13 \%$ for Cluster 3. Both centrifugation and washing caused an overall increase of Cluster $1 \quad(\mathrm{P}=0.047)$, whereas centrifugation decreased Cluster $2(\mathrm{P}=0.011)$. Only the addition of the oxidant affected significantly the subpopulation distribution (Fig. 3). In that case, Cluster 1 increased with time in the washed samples $(P=0.007)$, reaching $76.09 \pm 6.76 \%$ at $3 \mathrm{~h}$. Simultaneously, the oxidant decreased Cluster 3 proportion in washed samples $(P=0.003)$. Notably, the decrease of Cluster 3 at times 0 and $1 \mathrm{~h}$ was accompanied by an increase of Cluster 2, but at 2 and $3 \mathrm{~h}$ it was Cluster 1 that increased in turn (in each sample, the sum of the three subpopulations is $100 \%$, therefore a variation in one of them must be accompanied by variations in the other two). The decrease of Cluster 3 in presence of the oxidant was prevented by Trolox supplementation $(\mathrm{P}=0.002)$.

The analysis of sperm physiology by flow cytometry (Fig. 4) showed that nonviable spermatozoa $(\mathrm{PI}+)$ increased with time $(4.96 \pm 0.98$ per hour, $\mathrm{P}<0.001)$. Neither centrifugation nor washing affected death rate significantly, but the combination of washing and oxidative stress caused an immediate increase of PI+ spermatozoa $(14.29 \pm 3.07$ overall increase, $\mathrm{P}<0.001)$, but Trolox prevented this increase $(\mathrm{P}=0.008)$. Any treatment affected the percentage of YO-PRO-1spermatozoa ("nonapoptotic"), but the proportion of YO-PRO-1 + /PI-spermatozoa ("apoptotic") dropped almost to 0 in washed samples submitted to oxidative stress without Trolox, matching the increase of PI+ spermatozoa. The high- subpopulation remained stable with time $(\mathrm{P}=0.328)$, with a starting proportion of $78.03 \pm 3.90 \%$. Nevertheless, oxidative stress caused the loss of, but only in washed samples $(\mathrm{P}<0.001)$. In these samples, the proportion of high- spermatozoa dropped to $26.18 \pm 6.65 \%$ after only $1 \mathrm{~h}$, stabilizing around $9 \%$ afterward $(9.50 \pm 4.42 \%$ at $3 \mathrm{~h})$.

After $4 \mathrm{~h}$ of incubation, we detected a basal level of DNA-damaged cells of $22.30 \pm 3.52 \% \quad(\mathrm{P}<0.001)$, according to TUNEL labeling (Fig. 5). Any treatment increased TUNEL labeling, except when washed spermatozoa were incubated with oxidant $(67.94 \pm$ 

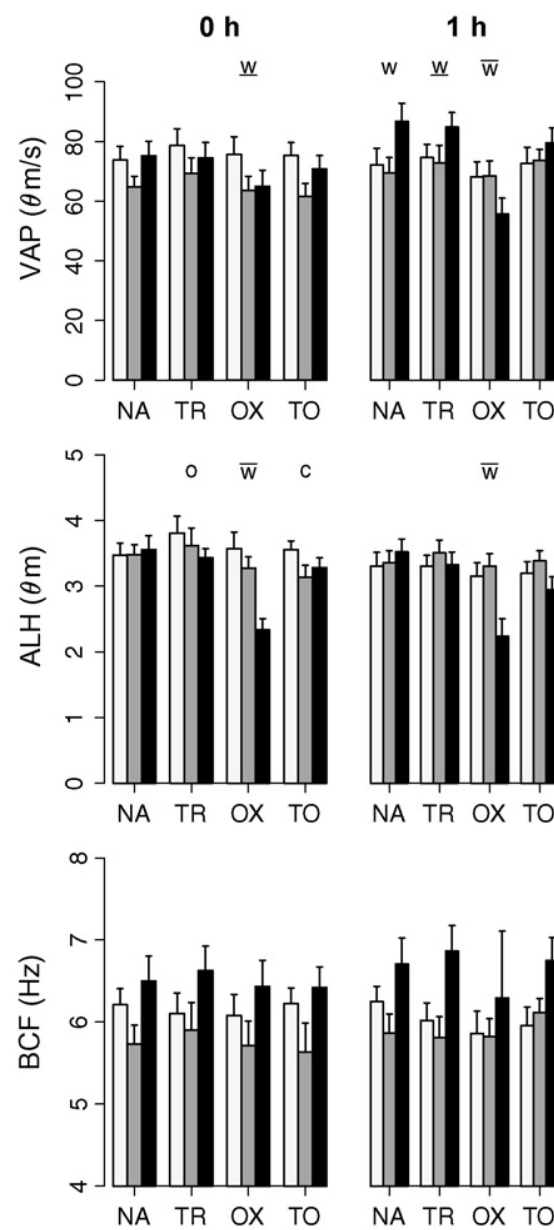

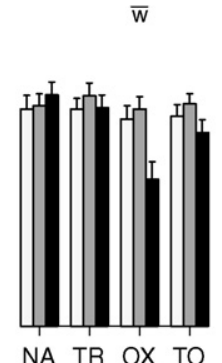

NA TR OX TO

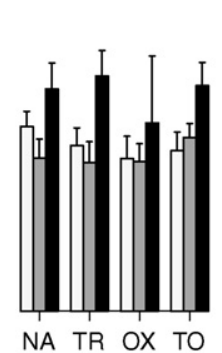

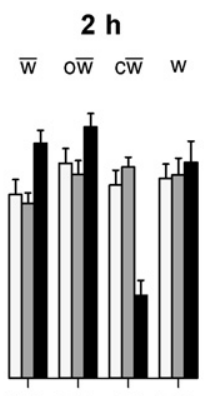

NA TR OX TO

$\overline{\mathrm{w}} \quad \mathrm{w}$

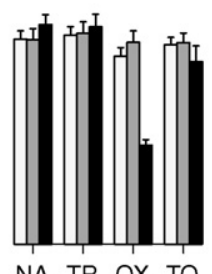

NA TR OX TO

$\overline{\mathrm{w}}$

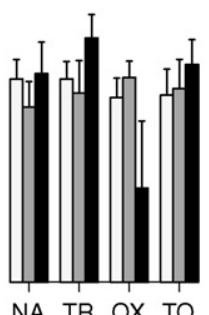

NA TR OX TO
$3 \mathrm{~h}$

$\overline{\mathrm{w}} \quad \underline{\mathrm{w}} \overline{\mathrm{w}} \quad \overline{\mathrm{w}}$

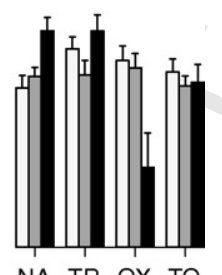

NA TR OX TO

$\overline{\mathrm{w}} \quad \overline{\mathrm{w}}$

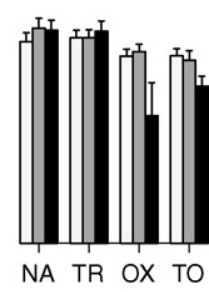

NA TR OX TO

$\bar{w} \quad w$

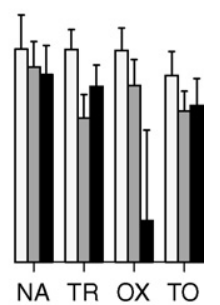

$\square$ Control

$\square$ Centrifugation

Washing

Fig. 2. Kinematic parameters (mean \pm SEM) at the four sampling times. VAP, average path velocity; ALH, head movement amplitude; BCF, beatcross frequency (only the most representative variables are shown; see text for a complete description). Means are shown for each supplement and treatment (NA, no additive; TR, $1 \mathrm{mM}$ Trolox; OX, $100 \mu \mathrm{M}$ Fe/1 mM ascorbate; TO, TR and OX). The letters indicate significant differences $(\mathrm{P}<0.05)$ within each time between the treatment Control/NS (first column on the left of each graph) and Control (o), Centrifuged (c), or Washing (w) treatments in each supplement group. An underline indicates $\mathrm{P}<0.01$, and an overbar indicates $\mathrm{P}<0.001$.

$5.07 \%$ of TUNEL+ cells, $\mathrm{P}<0.001)$. Nevertheless, Trolox prevented this increase of TUNEL+ spermatozoa $(\mathrm{P}=0.002)$.

Although the samples from the four males (Males 1 to 4) initially differed for most of the parameters $(\mathrm{P}<0.05)$, the effects of processing and supplements were generally not significantly different among them, suggesting a similar response between males. Nevertheless, we could detect some interactions between the male factor and the treatments and supplements. Thus, motility loss was larger for Male 4 when washed samples were challenged with the oxidant $(P=0.009)$, whereas Male 1 seemed to be more resilient to such a change $(\mathrm{P}=0.030)$. Similarly, Male 1 seemed to be less prone to decreasing VCL and ALH $(\mathrm{P}<0.05)$. It must be highlighted that Male 1 started with lower motility, velocity, and ALH than that of the others, which might be the cause for lower decreasing rates in these parameters. Contrarily, starting at similar values, Male 1 showed decreasing linearity and BCF with time, which was not evident in the other males. The between-male variability was lower for the physiologic parameters assessed by flow cytometry, even when considering starting values $(\mathrm{P}>0.1)$. However, TUNEL analysis showed a lower susceptibility for DNA damage in Males 2 and 3 after washing and oxidative stress (effect of-37.20 $\pm 12.76, \quad \mathrm{P}=0.005 \quad$ and $-25.97 \pm 12.76$, $\mathrm{P}=0.047$, respectively, compared with Male 1). 
Table 1

Kinematic parameters (mean \pm SEM) of the three sperm subpopulations isolated after the cluster analysis.

\begin{tabular}{lllll}
\hline Cluster & VAP $(\mu \mathrm{m} / \mathrm{sec})$ & STR $(\%)$ & ALH $(\mu)$ & BCF $(\mathrm{Hz})$ \\
\hline 1 & $22.70 \pm 0.30$ & $60.72 \pm 0.31$ & $1.95 \pm 0.02$ & $3.81 \pm 0.04$ \\
2 & $88.40 \pm 0.82$ & $88.79 \pm 0.19$ & $2.73 \pm 0.03$ & $8.69 \pm 0.04$ \\
3 & $95.09 \pm 0.96$ & $39.60 \pm 0.37$ & $5.11 \pm 0.06$ & $7.45 \pm 0.07$ \\
\hline
\end{tabular}

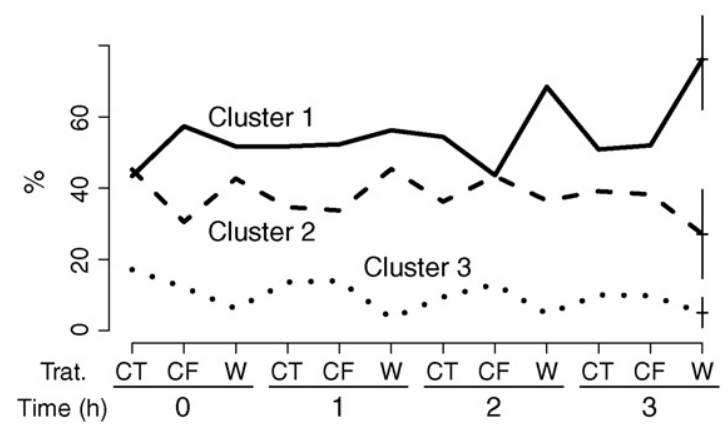

Fig. 3. Results of the subpopulation analysis (see subpopulation characteristics in Table 1) only for the samples supplemented with oxidant. Means of cluster proportions are shown for each sampling time and treatment (CT, Control; $\mathrm{CF}$, Centrifugation and redilution; W, washing). Notice how the proportion of the fast-erratic Cluster 3 decreases only for the washing treatment, with a corresponding increase of the fast-linear Cluster 2 (at 0 and $1 \mathrm{~h}$ ) or the slow Cluster 1 (at 2 and $3 \mathrm{~h}$ ). The vertical bar on the last point of each line shows the span of 2 standard errors for each cluster (error bars are not shown for Q8 all points to avoid clutter). Model significations are explained in the text (each cluster was analyzed independently).

\section{Discussion}

The results obtained in this study demonstrate that red deer spermatozoa are fairly resilient to centrifugation and washing, as noticed in other ungulates more than $30 \mathrm{yr}$ ago [34]. Our results agree with those of Varisli et al. [10], who submitted spermatozoa from mouse, rat, bull, ram, and boar to the mechanical stress caused by multiple pipetting. Whereas the motility of rat and mouse spermatozoa was severely affected by the mechanical stress, the samples from the ungulates were not significantly affected. In our study, submitting the spermatozoa to the mechanical stress caused by centrifugal forces and pellet packing had no important effects on any of the analyzed parameters, even after $3 \mathrm{~h}$ of incubation. It seems that, as proposed by Varisli et al. [10], the size and shape of the spermatozoon greatly influences its resistance to shear forces, including centrifugation. In fact, in the same study, These authors (and previously [9]) showed the susceptibility of rat spermatozoa to centrifugation, especially considering motility. This is not to say that centrifugation does not affect spermatozoa from ungulates but that the effect is small enough to be concealed by any beneficial outcome $[6,11,34]$. Spermatozoa from other species, but also smaller than rodent spermatozoa, also seem to resist centrifugation well [2].

However, sperm size alone does not fully explain the susceptibility to mechanical stress, as human spermatozoa seem to be prone to centrifugation damage $[5,7,8]$. It is possible that membrane composition or predisposition for osmotic shock could have a role in the loss of quality after centrifugation. In fact, we noticed a small overall negative effect of washing, but not centrifugation alone, on sperm motility. Therefore, the modification of the medium after pelleting caused that part of the spermatozoa to become immotile. In our study, we have used cryopreserved epididymal spermatozoa, therefore we can rule out any effect due to the removal of seminal plasma proteins. Other explanation that we can discard is that the loss of motility was caused by the generation of ROS, an event associated with mechanical stress in human semen $[12,15]$, as the antioxidant Trolox could not prevent this loss of motility, whereas it was effective when the oxidant was added to the medium. A likely explanation is that the removal of the remaining egg yolk and glycerol from the freezing extender would have osmotically challenged sensitive spermatozoa or have caused membrane changes conducive to loss of motility. Nevertheless, this loss of motility was relatively small, it did not increased with incubation time, and it did not reflect on the rest of parameters analyzed.

Therefore, we could not detect any effect of centrifugation, and the effect of washing was very limited. However, as soon as we added the oxidant, sperm quality of the washed samples decreased, whereas diluted or centrifuged samples remained largely unaffected. These results agree with our previous study [32], in which the effect of $\mathrm{Fe}$ in nonwashed samples was limited. We found that the Fe/ ascorbate system $(100 \mu \mathrm{M} \mathrm{Fe})$ readily increased intracellular ROS but that increase did not cause any change in sperm quality, except for an immediate but small decline of motility and a late ( $3 \mathrm{~h}$ at $37 \mathrm{C}$ ) increase of lipid peroxidation. This lack of effect was strikingly different than effects observed using hypoxanthine/ xanthine oxidase or hydrogen peroxide, which caused a quick drop of sperm motility and mitochondrial inactivation. It seems that thawed red deer spermatozoa are little affected by Fe/ascorbate (producing hydroxyl radical), provided that samples are simply diluted, and irrespective of being submitted to mechanical stresses 

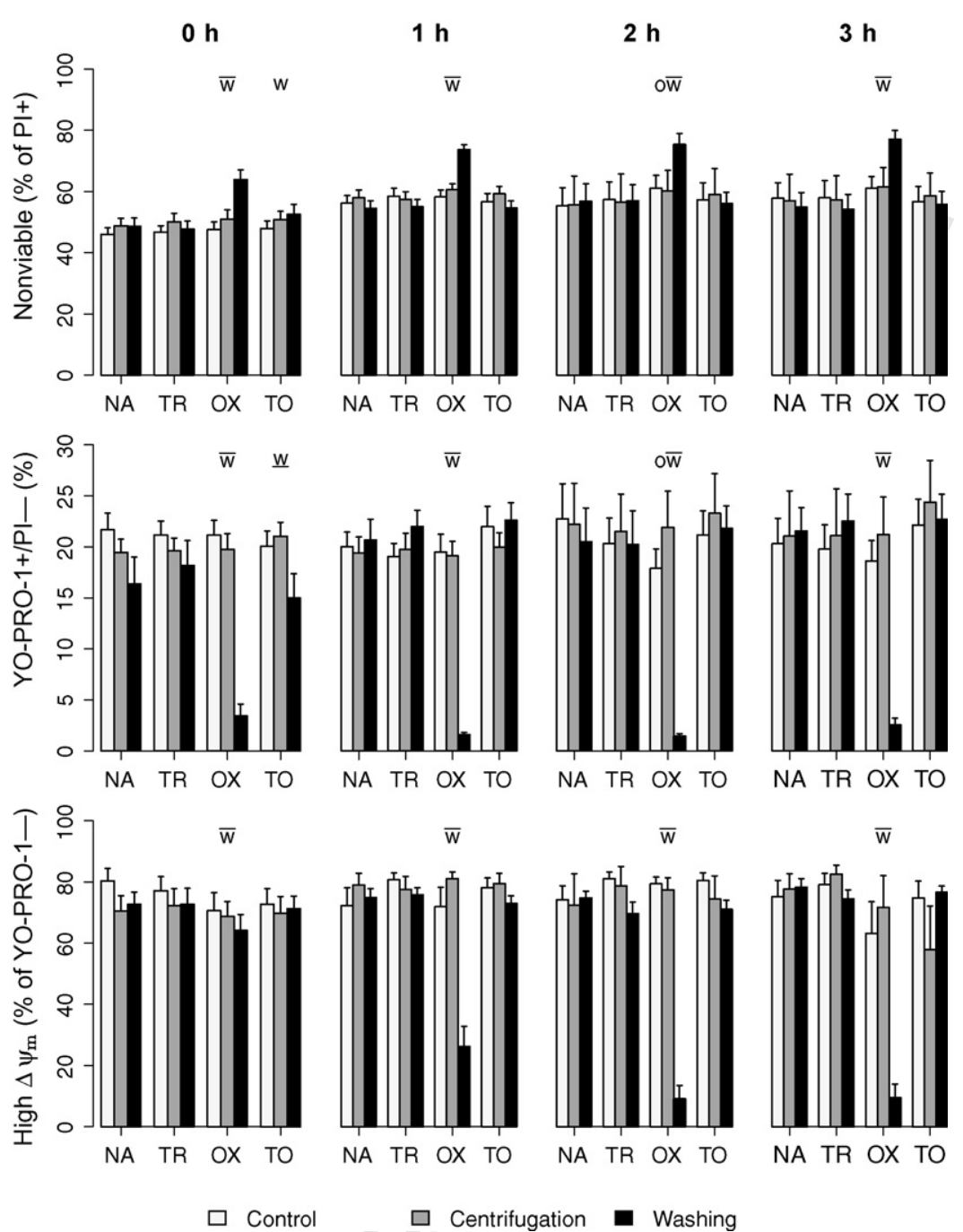

Fig. 4. Flow cytometry analyses at the four sampling times. Parameters (mean \pm SEM) are percentage of spermatozoa with damaged plasmalemma $(\mathrm{PI}+)$, percentage of YO-PRO-1 + /PI-spermatozoa ("apoptotic"), and percentage of the YO-PRO-1-subpopulation ("nonapoptotic") with active mitochondria (Mitotracker + ). Means are shown for each supplement and treatment (NA, no additive; TR, $1 \mathrm{mM} \mathrm{Trolox}$; OX, $100 \mu M$ Fe/1 mM ascorbate; TO, TR and OX). The letters indicate significant differences $(\mathrm{P}<0.05)$ within each time between the treatment Control/NA (first column on the left of each graph) and Control (o), Centrifuged (c), or Washing (w) treatments in each supplement group. An overbar indicates $\mathrm{P}<0.001$.

such as centrifugation. That contrasts with the human spermatozoon, which seems to be highly prone to lipoperoxidation and loss of motility in presence of $\mathrm{Fe}$ [35-37]. In fact, it has been shown that $\mathrm{Fe}$ is a potent promotor of lipid peroxidation in human spermatozoa in doses and times lower than those employed here [38]. In the current study, we did not assess lipid peroxidation, but our previous results ([32] and unpublished data) suggest that the incidence of Fe-induced lipoperoxidation would be minimal.

In contrast with control and centrifuged samples, washed samples submitted to oxidative stress showed an important decrease of motility, kinematic parameters, and mitochondrial activity with moderate decrease in the proportion of viable spermatozoa. This is the same kind of response we previously detected in thawed spermatozoa submitted to hydrogen peroxide [32]. It is not clear why washing increased the vulnerability of thawed spermatozoa to oxidative stress, as the samples had already been submitted to an important dilution with TALP-HEPES (to lower the sperm concentration from $200 \times 10 \mathrm{~mL}$ to $30 \times 10 \mathrm{~mL}$ ). The most likely hypothesis is that the change of medium caused some kind of alteration in the plasma membranes. The diluted egg yolk in the sample ( $\sim 3 \%$ after the initial dilution) could still have had a protective function on sperm 


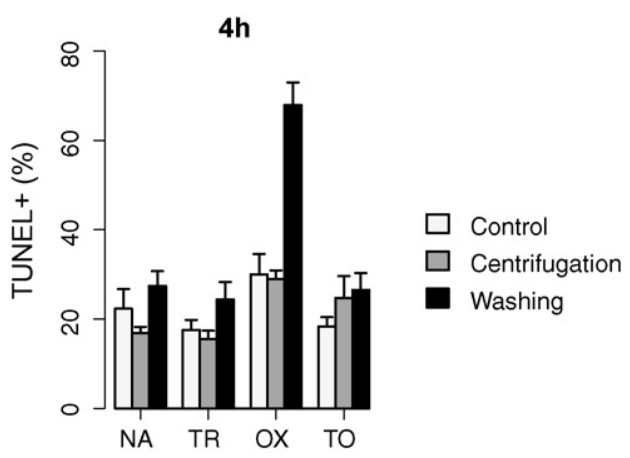

Fig. 5. TUNEL analysis after $4 \mathrm{~h}$ of incubation. Means are shown for each supplement and treatment (NA, no additive; TR, $1 \mathrm{mM}$ Trolox; OX, $100 \mu \mathrm{M} \mathrm{Fe} / 1 \mathrm{mM}$ ascorbate; TO, TR and OX). The oxidant considerably increased the percentage of TUNEL-positive spermatozoa $(\mathrm{P}<0.001)$ only in washed samples, but this effect was completely abolished by the antioxidant.

membranes [39], or, maybe, the combination of centrifugation and medium change removed factors from the sperm membrane, making the cells more vulnerable to oxidative stress. The antioxidant supplementation prevented the action of the oxidant in the washed samples. Trolox is a soluble form of vitamin $\mathrm{E}$ ( $\alpha$-tocopherol), successfully used for protecting spermatozoa during storage or cryopreservation $[40,41]$. In human spermatozoa, supplementing the media with antioxidants ameliorated the sperm quality after techniques involving centrifugation [17-19]. In our case, Trolox neutralized totally or in part, depending on the studied parameter, the effect of the oxidative stress. More importantly, it prevented the increase of DNA damage, assessed as the percentage of TUNEL+ cells. Whereas Fe increased the proportion of positive cells considerably, there was no difference between the samples treated with Trolox and the control, irrespective of the treatment. It must be taken into account that the effect of the antioxidant might not only be directly exerted by scavenging free radicals but can also be indirect, helping to maintain and recover the normal distribution of antioxidant enzymes [42].

Thus, the dramatic effect of induced oxidative stress on washed samples indicates that such a treatment increases considerably the susceptibility of red deer sperm samples to the oxidative insult. However, the results in absence of induced oxidative stress suggest that, as our samples seem little prone to trigger ROS production after centrifugation stress, this problem might be a minor concern in artificial reproductive techniques. Conversely, notable ROS production has been described for human spermatozoa in different experimental conditions [14,43-45], including prepara- tion techniques such as washing, density gradient centrifugation, or swim-up [46,47]. Moreover, Chi et al. [48] detected increasing ROS levels after washing human spermatozoa, and when antioxidants were added to the washing media, that effect was ameliorated. As noted previously, in the current study and in previous reports [31,32] we could not detect differences among red deer samples treated or not treated with antioxidants unless we added oxidative agents.

The differences between males suggest a differential response to oxidative stress depending on individual factors. Whereas feeding or body condition surely influences sperm ability to sustain oxidative stress (through changes in membrane composition, availability of vitamins, and expression of antioxidant enzymes), genetic variation cannot be ruled out (as it might be the basis for differences on motility patterns [49] or cryopreservation ability [50]). In fact, one of the advantages of working with wild populations is the high individual variability often found. Here, we have shown that the susceptibility to oxidative stress after washing was common to the four males analyzed, but there were important differences on how that susceptibility was expressed. The differences found in the different levels of DNA damage highlight the need for improving the sperm work techniques to prevent damage to samples from susceptible males, as thoroughly tested protocols might still be detrimental to these samples, spoiling genetically important germplasm.

It is interesting to consider our results regarding the subpopulation analysis of the motility data. Because the proportions of the three subpopulations studied did not differ significantly across times or treatments (centrifugation or washing), we propose that the internal structure of the samples remained stable, despite the decrease of total motility. Therefore, the oxidative stress not only lowered the percentage of motile spermatozoa in the washed samples but also disrupted their subpopulation pattern (which we observed previously during the cryopreservation protocol [51]). However, the alteration of the subpopulation pattern was most evident at longer incubation times ( 2 and $3 \mathrm{~h}$ ), which might be associated with the loss of mitochondrial activity, as noted previously [31].

In conclusion, we have found that centrifugation alone seems to have little effect on the quality of thawed deer spermatozoa, but washing incrementally increased their susceptibility to exogenous oxidative stress. Our results suggest a higher resilience of deer spermatozoa to procedures involving centrifugation, which is very different than the results of studies on rodents or human spermatozoa. Although our results suggest that damage 
due to oxidative stress after washing could be a minor concern in red deer spermatozoa, we propose the addition of simple antioxidants to the manipulation media, as they could protect thawed spermatozoa against eventual oxidative stress during routine procedures in other experimental settings (different media, longer incubation times, etc.). In fact, one of our most important findings is the capacity of the antioxidant treatment preventing DNA damage in washed samples. It must be taken into account that DNA damage, contrary to our experiment, can also occur without a corresponding alteration in sperm motility [52], being thus unnoticed, and making it necessary to provide protective measures during sperm manipulation. Moreover, male-to-male variability concerning the resilience of spermatozoa to oxidative stress after washing, including DNA damage, was clearly detectable. Use of an antioxidant could prevent a mild oxidative stress from spoiling a susceptible sample during preparation. Moreover, we used thawed samples from the epididymis, whereas treating ejaculated semen might be more risky, as washing involves removing seminal plasma. Several studies on small ruminants have highlighted the importance of seminal plasma to the maintenance of membrane stability and to the integrity of the antioxidant system of the spermatozoa. Therefore, use of antioxidants in preparation media could be even more important in these cases and should be thoroughly explored.

\section{Acknowledgments}

This work was supported by the Spanish Ministry of Science and Innovation (grant no. AGL2004-05904/ GAN) and by the Education and Science Council of Junta de Comunidades de Castilla-La Mancha (grant no. PAC06-0047). The authors thank Enrique del Olmo, Alfonso Bisbal, and José Luis Ros for help with the experiments. A.E. Domínguez-Rebolledo was supported by Consejo Nacional de Ciencia y Tecnología (CONACyT, Mexico). F. Martìnez-Pastor was supported by the Juan de la Cierva program and by the Ramón y Cajal program (Ministry of Science and Innovation, Spain), and M.R. Fernández-Santos was supported by the Juan de la Cierva program (Ministry of Science and Innovation, Spain).

\section{References}

[1] Agca Y, Critser JK. Cryopreservation of spermatozoa in assisted reproduction. Semin Reprod Med 2002;20:15-23.

[2] Rijsselaere T, Van Soom A, Maes D, de Kruif A. Effect of centrifugation on in vitro survival of fresh diluted canine spermatozoa. Theriogenology 2002;57:1669-81.
[3] Lymberopoulos A, Khalifa T. Sperm chromatin stability during in vitro manipulation of beef bull semen. Reprod Domest Anim 2008 .

[4] Okazaki T, Abe S, Yoshida S, Shimada M. Seminal plasma damages sperm during cryopreservation, but its presence during thawing improves semen quality and conception rates in boars with poor post-thaw semen quality. Theriogenology 2009;71:491-8.

[5] Sharma RK, Vemulapalli S, Kohn S, Agarwal A. Effect of centrifuge speed, refrigeration medium, and sperm washing medium on cryopreserved sperm quality after thawing. Arch Androl 1997;39:33-8.

[6] Gil J, Soderquist L, Rodriguez-Martinez H. Influence of centrifugation and different extenders on post-thaw sperm quality of ram semen. Theriogenology 2000;54:93-108.

[7] Ng SC, Bongso TA, Sathananthan H, Tok VC, Ratnam SS. Micro-centrifugation of human spermatozoa: its effect on fertilization of hamster oocytes after micro-insemination spermatozoal transfer. Hum Reprod 1990;5:209-11.

[8] Alvarez JG, Lasso JL, Blasco L, Nunez RC, Heyner S, Caballero PP, Storey BT. Centrifugation of human spermatozoa induces sublethal damage; separation of human spermatozoa from seminal plasma by a dextran swim-up procedure without centrifugation extends their motile lifetime. Hum Reprod 1993;8:1087-92.

[9] Katkov II, Mazur P. Influence of centrifugation regimes on motility, yield, and cell associations of mouse spermatozoa. J Androl 1998; 19:232-41.

[10] Varisli O, Uguz C, Agca C, Agca Y. Various physical stress factors on rat sperm motility, integrity of acrosome, and plasma membrane. J Androl 2009;30:75-86.

[11] Carvajal G, Cuello C, Ruiz M, Vazquez JM, Martinez EA, Roca J. Effects of centrifugation before freezing on boar sperm cryosurvival. J Androl 2004;25:389-96.

[12] Aitken RJ, Clarkson JS. Significance of reactive oxygen species and antioxidants in defining the efficacy of sperm preparation techniques. J Androl 1988;9:367-76.

[13] Iwasaki A, Gagnon C. Formation of reactive oxygen species in spermatozoa of infertile patients. Fertil Steril 1992;57:409-16.

[14] Twigg J, Irvine DS, Houston P, Fulton N, Michael L, Aitken RJ. Iatrogenic DNA damage induced in human spermatozoa during sperm preparation: protective significance of seminal plasma. Mol Hum Reprod 1998;4:439-45.

[15] de Jager C, Bornman MS, Aneck-Hahn NH, du Toit D, Viljoen E. Effect of rotation on the generation of reactive oxygen species in human semen. Andrologia 1996;28:291-3.

[16] Rodriguez-Gil JE, Rigau T. Effects of slight agitation on the quality of refrigerated boar sperm. Anim Reprod Sci 1995;39: $141-6$.

[17] Griveau JF, Le Lannou D. Effects of antioxidants on human sperm preparation techniques. Int J Androl 1994;17:225-31.

[18] Parinaud J, Le Lannou D, Vieitez G, Griveau JF, Milhet P, Richoilley G. Enhancement of motility by treating spermatozoa with an antioxidant solution (Sperm-Fit) following ejaculation. Hum Reprod 1997;12:2434-6.

[19] Hughes CM, Lewis SE, McKelvey-Martin VJ, Thompson W. The effects of antioxidant supplementation during Percoll preparation on human sperm DNA integrity. Hum Reprod 1998;13: 1240-7.

[20] Cseh S, Solti L. Importance of assisted reproductive technologies in the conservation of wild, rare or indigenous ungulates: review article. Acta Vet Hung 2000;48:313-23.

[21] Fletcher TJ. Farmed deer: new domestic animals defined by controlled breeding. Reprod Fertil Dev 2001;13:511-6. 
[22] Martinez-Pastor F, Anel L, Guerra C, Alvarez M, Soler AJ, Garde JJ, et al. Seminal plasma improves cryopreservation of Iberian red deer epididymal sperm. Theriogenology 2006;66: 1847-56.

[23] Fernandez-Santos M, Esteso M, Montoro V, Soler A, Garde J. Cryopreservation of Iberian red deer (Cervus elaphus hispanicus) epididymal spermatozoa: effects of egg yolk, glycerol and cooling rate. Theriogenology 2006;66:1931-42.

[24] Fernandez-Santos MR, Esteso MC, Montoro V, Soler AJ, Garde JJ. Influence of various permeating cryoprotectants on freezability of Iberian red deer (Cervus elaphus hispanicus) epididymal spermatozoa: effects of concentration and temperature of addition. J Androl 2006;27:734-45.

[25] Fernandez-Santos MR, Martinez-Pastor F, Garcia-Macias V, Esteso MC, Soler AJ, de Paz P, et al. Extender osmolality and sugar supplementation exert a complex effect on the cryopreservation of Iberian red deer (Cervus elaphus hispanicus) epididymal spermatozoa. Theriogenology 2007;67:738-53.

[26] Garde J, Martinez-Pastor F, Gomendio M, Malo A, Soler A, Fernandez-Santos $\mathrm{M}$, et al. The application of reproductive technologies to natural populations of red deer. Reprod Domest Anim 2006;41(Suppl 2):93-102.

[27] Martinez-Pastor F, Martinez F, Garcia-Macias V, Esteso M, Anel E, Fernandez-Santos M, et al. A pilot study on post-thawing quality of Iberian red deer spermatozoa (epididymal and electroejaculated) depending on glycerol concentration and extender osmolality. Theriogenology 2006;66:1165-72.

[28] Martinez-Pastor F, Martinez F, Alvarez M, Maroto-Morales A, Garcia-Alvarez O, Soler AJ, et al. Cryopreservation of Iberian red deer (Cervus elaphus hispanicus) spermatozoa obtained by electroejaculation. Theriogenology 2009;71:628-38.

[29] Roca J, Hernandez M, Carvajal G, Vazquez JM, Martinez EA Factors influencing boar sperm cryosurvival. J Anim Sci 2006;84:2692-9.

[30] Mortimer D, Serres C, Mortimer ST, Jouannet P. Influence of image sampling frequency on the perceived movement characteristics of progressively motile human spermatozoa. Gamete Res 1988;20:313-27.

[31] Martinez-Pastor F, Fernandez-Santos MR, del Olmo E, Dominguez-Rebolledo AE, Esteso MC, Montoro V, Garde JJ. Mitochondrial activity and forward scatter vary in necrotic, apoptotic and membrane-intact spermatozoan subpopulations. Reprod Fertil Dev 2008;20:547-56.

[32] Martinez-Pastor F, Aisen E, Fernandez-Santos MR, Esteso MC, Maroto-Morales A, Garcia-Alvarez O, Garde JJ. Reactive oxygen species generators affect quality parameters and apoptosis markers differently in red deer spermatozoa. Reproduction 2009; 137:225-35.

[33] R Development Core Team. R: A Language and Environment for Statistical Computing. R Foundation for Statistical Computing, 2007.

[34] Pickett BW, Sullivan JJ, Byers WW, Pace MM, Remmenga EE. Effect of centrifugation and seminal plasma on motility and fertility of stallion and bull spermatozoa. Fertil Steril 1975;26: 167-74.

[35] Aitken RJ, Harkiss D, Buckingham DW. Analysis of lipid peroxidation mechanisms in human spermatozoa. Mol Reprod Dev 1993;35:302-15.

[36] Storey BT. Biochemistry of the induction and prevention of lipoperoxidative damage in human spermatozoa. Mol Hum Reprod 1997;3:203-13.

[37] Huang YL, Tseng WC, Lin TH. In vitro effects of metal ions (Fe, $\mathrm{Mn}, \mathrm{Pb}$ ) on sperm motility and lipid peroxidation in human semen. J Toxicol Environ Health A 2001;62:259-67.
[38] Aitken RJ, Wingate JK, De Iuliis GN, McLaughlin EA. Analysis of lipid peroxidation in human spermatozoa using BODIPY C11. Mol Hum Reprod 2007;13:203-11.

[39] Bergeron A, Manjunath P. New insights towards understanding the mechanisms of sperm protection by egg yolk and milk. Mol Reprod Dev 2006;73:1338-44.

[40] Ball BA, Medina V, Gravance CG, Baumbe J. Effect of antioxidants on preservation of motility,viability and acrosomal integrity of equine spermatozoa during storage at 5 degrees C. Theriogenology 2001;56:577-89.

[41] Peña F, Johannisson A, Wallgren M, Rodriguez Martinez H. Antioxidant supplementation in vitro improves boar sperm motility and mitochondrial membrane potential after cryopreservation of different fractions of the ejaculate. Anim Reprod Sci 2003;78:85-98.

[42] Marti E, Marti JI, Muino-Blanco T, Cebrian-Perez JA. Effect of the cryopreservation process on the activity and immunolocalization of antioxidant enzymes in ram spermatozoa. J Androl 2008;29:459-67.

[43] Aitken J, Krausz C, Buckingham D. Relationships between biochemical markers for residual sperm cytoplasm, reactive oxygen species generation, and the presence of leukocytes and precursor germ cells in human sperm suspensions. Mol Reprod Dev 1994;39:268-79.

[44] Aitken RJ, Fisher HM, Fulton N, Gomez E, Knox W, Lewis B, Irvine $\mathrm{S}$. Reactive oxygen species generation by human spermatozoa is induced by exogenous NADPH and inhibited by the flavoprotein inhibitors diphenylene iodonium and quinacrine. Mol Reprod Dev 1997;47:468-82.

[45] Aitken RJ, Wingate JK, De Iuliis GN, Koppers AJ, McLaughlin EA. Cis-unsaturated fatty acids stimulate reactive oxygen species generation and lipid peroxidation in human spermatozoa. $\mathbf{J}$ Clin Endocrinol Metab 2006;91:4154-63.

[46] Agarwal A, Ikemoto I, Loughlin KR. Effect of sperm washing on levels of reactive oxygen species in semen. Arch Androl 1994;33: 157-62.

[47] Allamaneni S, Agarwal A, Nallella K, Sharma R, Thomas A, Sikka S. Characterization of oxidative stress status by evaluation of reactive oxygen species levels in whole semen and isolated spermatozoa. Fertil Steril 2005;83:800-3.

[48] Chi HJ, Kim JH, Ryu CS, Lee JY, Park JS, Chung DY, et al. Protective effect of antioxidant supplementation in sperm-preparation medium against oxidative stress in human spermatozoa. Hum Reprod 2008;23:1023-8.

[49] Quintero-Moreno A, Rigau T, Rodriguez-Gil J. Multivariate cluster analysis regression procedures as tools to identify motile sperm subpopulations in rabbit semen and to predict semen fertility and litter size. Reprod Domest Anim 2007;42: 312-9.

[50] Thurston LM, Siggins K, Mileham AJ, Watson PF, Holt WV. Identification of amplified restriction fragment length polymorphism markers linked to genes controlling boar sperm viability following cryopreservation. Biol Reprod 2002;66:545-54.

[51] Martinez-Pastor F, Garcia-Macias V, Alvarez M, Herraez P, Anel L, de Paz P. Sperm subpopulations in Iberian red deer epididymal sperm and their changes through the cryopreservation process. Biol Reprod 2005;72:316-27.

[52] Twigg J, Fulton N, Gomez E, Irvine D, Aitken R. Analysis of the impact of intracellular reactive oxygen species generation on the structural and functional integrity of human spermatozoa: lipid peroxidation, DNA fragmentation and effectiveness of antioxidants. Hum Reprod 1998;13:1429-36. 